\title{
The F-theory geometry with most flux vacua
}

\author{
Washington Taylor and Yi-Nan Wang \\ Center for Theoretical Physics, Department of Physics, Massachusetts Institute of Technology, \\ 77 Massachusetts Avenue, Cambridge, MA 02139, U.S.A. \\ E-mail: wati@mit.edu, wangyn@mit.edu
}

Abstract: Applying the Ashok-Denef-Douglas estimation method to elliptic Calabi-Yau fourfolds suggests that a single elliptic fourfold $\mathcal{M}_{\text {max }}$ gives rise to $\mathcal{O}\left(10^{272,000}\right)$ F-theory flux vacua, and that the sum total of the numbers of flux vacua from all other F-theory geometries is suppressed by a relative factor of $\mathcal{O}\left(10^{-3000}\right)$. The fourfold $\mathcal{M}_{\text {max }}$ arises from a generic elliptic fibration over a specific toric threefold base $B_{\max }$, and gives a geometrically non-Higgsable gauge group of $E_{8}^{9} \times F_{4}^{8} \times\left(G_{2} \times \mathrm{SU}(2)\right)^{16}$, of which we expect some factors to be broken by G-flux to smaller groups. It is not possible to tune an $\mathrm{SU}(5) \mathrm{GUT}$ group on any further divisors in $\mathcal{M}_{\max }$, or even an $\mathrm{SU}(2)$ or $\mathrm{SU}(3)$, so the standard model gauge group appears to arise in this context only from a broken $E_{8}$ factor. The results of this paper can either be interpreted as providing a framework for predicting how the standard model arises most naturally in F-theory and the types of dark matter to be found in a typical F-theory compactification, or as a challenge to string theorists to explain why other choices of vacua are not exponentially unlikely compared to F-theory compactifications on $\mathcal{M}_{\max }$.

KEYwords: Flux compactifications, F-Theory, Superstring Vacua

ARXIV EPRINT: 1511.03209 


\section{Contents}

1 Introduction 1

2 F-theory on the fourfold $\mathcal{M}_{\max } \quad 2$

2.1 The geometry of $\mathcal{M}_{\max }$ as an elliptic fibration 3

2.2 Geometric non-Higgsable structures on $\mathcal{M}_{\max } \quad 4$

2.3 The standard model and dark matter on $\mathcal{M}_{\max }$

3 Distribution of flux vacua $\quad 6$

3.1 Flux vacua on $\mathcal{M}_{\max } \quad 6$

3.2 Suppression of other F-theory compactifications 8

$\begin{array}{lll}3.3 & \text { Other threefolds that are } B_{2} \text { bundles over } \mathbb{P}^{1} & 10\end{array}$

4 Possible flaws in this scenario $\quad 13$

$\begin{array}{ll}\text { A Linear transformation of the polytope containing } \mathcal{M}_{\max } & 16\end{array}$

B The Weierstrass model on $B_{\max }$ and the possibility of tuning 16

\section{Introduction}

The apparent existence of an enormous number of possible consistent $4 \mathrm{~d}$ vacuum solutions to string theory poses practical and philosophical challenges for the predictive power of the theory. On one hand, Weinberg's argument [1] and the possibility of cosmological inflation and vacuum tunneling in a multiverse fit naturally with the many vacua of string theory into an anthropic explanation for the observation of a small but nonzero cosmological constant, roughly $10^{-120}$ in natural units [2]. On the other hand, there is as yet no sound methodology for computing the relative abundance of different string vacuum solutions, and we are far from a complete understanding of the full set of possible vacuum solutions with supersymmetry, let alone of those without supersymmetry.

The largest numbers of different string vacua studied to date arise in the form of "flux compactifications" [3-5]. A flux compactification is a string compactification on a geometric space $\mathcal{M}$, combined with a choice of generalized $p$-form fluxes, analogous to magnetic flux, that thread various topological cycles on $\mathcal{M}$. In general, the set of fluxes is constrained by a tadpole condition (e.g., from varying one of the fields in the Lagrangian in a supergravity approximation) so that the number of flux vacua that can arise for any given geometry becomes bounded, though it can be exponentially large. For type IIB string theory, the number of flux compactifications on certain geometries is famously estimated at $\sim \mathcal{O}\left(10^{500}\right)$. For type IIA string theory there may be infinite families of 
flux vacua [6], though there are believed to be only a finite number of possibilities at any given compactification scale [7]. The largest concrete numbers of flux vacua known arise in F-theory [8-10], a nonperturbative version of type IIB theory with an axiodilaton that is allowed to vary over the compact manifold, which is a complex threefold (real 6-manifold) in the case of compactification to $4 \mathrm{~d}$.

In this paper we argue that virtually all F-theory flux compactifications may arise from a single geometry, $\mathcal{M}_{\max }$, and that all other F-theory flux compactifications taken together may represent a fraction of $\sim \mathcal{O}\left(10^{-3000}\right)$ of the total set. The geometry $\mathcal{M}_{\text {max }}$, which was first identified in [11, 12], is an elliptically fibered Calabi-Yau fourfold with Hodge numbers $h^{1,1}=252, h^{3,1}=303,148 . \mathcal{M}_{\max }$ can be understood as a generic elliptic fibration (i.e., arising from a generic Weierstrass model describing the axiodilaton) over a particular base $B_{\max }$, which is a complex threefold with a simple description in toric geometry. The identification of the base $B_{\max }$ gives a great deal of information about the possible forms of the low-energy $4 \mathrm{~d}$ physics that can arise from an F-theory flux compactification on $B_{\max }$. In particular, the geometric structure of $4 \mathrm{~d}$ "non-Higgsable clusters" [13-17] on $B_{\max }$ shows that in the absence of fluxes and an associated superpotential, the gauge group of the low-energy $4 \mathrm{~d}$ theory at a generic point in the moduli space of $\mathcal{M}_{\max }$ would be

$$
G_{\max }=E_{8}^{9} \times F_{4}^{8} \times\left(G_{2} \times \mathrm{SU}(2)\right)^{16},
$$

with charged matter transforming in the bifundamental representation of each $G_{2} \times \mathrm{SU}(2)$ factor. The presence of flux (G-flux) can, and likely will in most flux vacua, break some or all of these factors into subgroups in a fashion amenable to statistical analysis on the large set of possible fluxes.

Thus, it seems natural to speculate that the multiverse could be dominated by Ftheory flux vacua on $\mathcal{M}_{\max }$, among which a typical vacuum would have as gauge group a subgroup of $G_{\max }$. The standard model could be realized in this scenario through breaking of an $E_{8}$ sector to $\mathrm{SU}(3) \times \mathrm{SU}(2) \times \mathrm{U}(1)$, with the remaining factors (or subgroups thereof) of $G_{\max }$ providing a set of hidden dark matter sectors, connecting to the standard model only through gravitational and nonperturbative physics. If this story, or some modified version thereof, is correct, it provides a predictive framework for string theory despite the plethora of vacuum solutions.

In section 2 we describe the geometry of $\mathcal{M}_{\max }$ in more detail. In section 3 , we describe the possible physics of a typical F-theory on $\mathcal{M}_{\max }$ vacuum, and discuss the relative numbers of vacua associated with other compactification geometries. In section 4, we describe some of the many possible ways in which this nice story may break down.

\section{F-theory on the fourfold $\mathcal{M}_{\max }$}

In this section we give a description of the fourfold $\mathcal{M}_{\max }$ and the corresponding F-theory models. The notation used follows that of [18], where a more detailed description is given of toric F-theory compactifications of this type. For more general background on F-theory and compactifications, see [5, 19, 20]. 


\subsection{The geometry of $\mathcal{M}_{\max }$ as an elliptic fibration}

The complex four-manifold $\mathcal{M}_{\max }$ is an elliptically fibered Calabi-Yau manifold with Hodge numbers and fourth Betti number

$$
\begin{aligned}
h^{1,1} & =252 \\
h^{2,1} & =0 \\
h^{3,1} & =303,148 \\
h^{2,2} & =4\left(h^{1,1}+h^{3,1}\right)+44-2 h^{2,1}=1,213,644 \\
b_{4} & =2+2 h^{3,1}+h^{2,2}=1,819,942 .
\end{aligned}
$$

The Euler character is thus $\chi=6\left(8+h^{1,1}+h^{3,1}-h^{2,1}\right)=1,820,448$. This geometry was identified in $[11,12]$ using toric and Landau-Ginzburg model approaches to constructing Calabi-Yau fourfolds. The four-manifold $\mathcal{M}_{\max }$ is the Calabi-Yau fourfold with the largest known value of $h^{3,1}$. This fourfold and its mirror share the largest known Euler character, and the potential importance of these manifolds for F-theory was commented on in [21].

The geometry of $\mathcal{M}_{\max }$ can be understood as that of a generic elliptic fibration over a complex threefold base $B_{\max } . \quad B_{\max }$ is itself formed as a $B_{2}$ bundle over $\mathbb{P}^{1}$, where $B_{2}$ is a toric surface characterized by a closed cycle of toric divisors (curves, corresponding to rays in the toric fan) with self-intersections $0,+6,-12 / /-11 / /-12 / /-12 / /-12 / /-12 / /$ $-12 / /-12 / /-12$, where // denotes the sequence of self-intersections $-1,-2,-2,-3,-1,-5$, $-1,-3,-2,-2,-1$. $\quad B_{2}$ itself supports a generic elliptic Calabi-Yau threefold that has Hodge numbers $(251,251)[22,23]$. In the language of toric geometry [24, 25], $B_{2}$ is described by a $2 \mathrm{D}$ fan generated by a set of rays $v_{i} \in N=\mathbb{Z}^{2}$. The rays $v_{i}$ can be taken to be

$$
\begin{aligned}
v_{1} & =(-1,-12) \\
v_{2} & =(0,1) \\
v_{3}= & (1,6) \\
\vdots & \vdots \\
v_{99}= & (0,-1) .
\end{aligned}
$$

The intermediate rays can be determined by the condition $v_{i-1}+v_{i+1}+\left(C_{i} \cdot C_{i}\right) v_{i}=0$, where $C_{i} \cdot C_{i}$ is the self-intersection of the $i$ th curve. From the rays $v_{i}$ we can construct the toric fan for $B_{\max }$, which is given by the rays

$$
\begin{aligned}
w_{0} & =(0,0,1) \\
w_{i} & =\left(v_{i}, 0\right), \quad 1 \leq i \leq 99 \\
w_{100} & =(84,492,-1)=\left(12 v_{15},-1\right),
\end{aligned}
$$

where $C_{15}$ is the curve in $B_{2}$ of self-intersection -11 . The $3 \mathrm{D}$ cones of the fan for $B_{\max }$ are spanned by $\left(w_{0}, w_{i}, w_{i+1}\right)$ and $\left(w_{100}, w_{i}, w_{i+1}\right)$, including the cyclic case $\left(w_{0} / w_{100}, w_{99}, w_{1}\right)$. This manifestly gives $B_{\max }$ the structure of a $B_{2}$ bundle over $\mathbb{P}^{1}$, where the toric projection onto the third axis corresponds to the fibration structure. The "twist" in this bundle is 
characterized by the offset $12 v_{15}$ of the ray $w_{100}$. The $3 \mathrm{D}$ polytope containing the vertices $w_{i}$ is defined by the tetrahedron spanned by $w_{0}, w_{1}, w_{2}, w_{100}$.

A direct toric computation of the number of monomials in the generic Weierstrass model $y^{2}=x^{3}+f x+g$ along the lines of [14, 17, 26], with an appropriate offset for automorphisms, reproduces the value (2.3). Alternatively, constructing the generic elliptic fibration over $B_{\max }$ as a hypersurface in a toric variety by extending the polytope for $B_{\max }$ to the polytope of a $\mathbb{P}^{2,3,1}$ fibration over $B_{\max }$ gives a construction that is linearly equivalent to that described in [27]. For the details of the linear transformation see appendix A.

\subsection{Geometric non-Higgsable structures on $\mathcal{M}_{\max }$}

A useful tool in classifying geometries of base manifolds that support elliptic fibrations for F-theory is the "non-Higgsable" geometric structure of the base. Non-Higgsable clusters refer to gauge groups or products of gauge groups that arise from connected sets of codimension one Kodaira singularities in generic elliptic fibrations over a given base. NonHiggsable clusters for base surfaces were classified in [13] and have provided a valuable tool in classifying elliptic threefolds and associated 6d supergravity theories [22, 23, 28-30] as well as $6 \mathrm{~d}$ superconformal field theories [31-33]. Non-Higgsable clusters for base threefolds were systematically analyzed in [16] and have been used in analyzing 4d F-theory models in $[14,15,17,18]$. For the base $B_{\max }$ the analysis of monomials in $f, g$ and the associated discriminant $\Delta=4 f^{3}+27 g^{2}$ in the Weierstrass model shows that there are non-Higgsable gauge factors on precisely the divisors associated with curves in $B_{2}$ that carry non-Higgsable gauge factors in the corresponding $6 \mathrm{~d}$ theory, where the -12 (and -11 ) curves carry $E_{8}$ factors, the -5 curves carry $F_{4}$ factors, and the $-3,-2,-2$ sequences each carry $G_{2} \times \mathrm{SU}(2)$ products with bifundamental matter. Thus, the geometrically non-Higgsable gauge group of a generic elliptic fibration over $B_{\max }$ is

$$
G_{\max }=E_{8}^{9} \times F_{4}^{8} \times\left(G_{2} \times \mathrm{SU}(2)\right)^{16} .
$$

This group was originally associated with the elliptic Calabi-Yau fourfold $\mathcal{M}_{\max }$ in [11] using the method of "tops," which describe both Higgsable and non-Higgsable gauge group factors.

While for F-theory compactifications to six dimensions, there is a precise correspondence between geometrically non-Higgsable structure and massless gauge groups and matter in the low-energy theory, the connection is less transparent for $4 \mathrm{~d}$ compactifications. In particular, as discussed in [14-16], flux on seven-brane world-volumes can break geometrically non-Higgsable gauge factors, and G-flux produces a superpotential that can in principle drive the theory to loci with a further enhanced gauge symmetry. In the case of $B_{\max }$, however, there is no possibility of acquiring further gauge symmetry without producing codimension two singularities where $(f, g, \Delta)$ vanish to orders $(4,6,12)$, so it seems impossible to produce additional nonabelian gauge groups (such as a GUT SU(5) or standard model $\mathrm{SU}(3) \times \mathrm{SU}(2))$ by tuning Weierstrass moduli, either by hand or through forcing by a superpotential, without changing the base or a hitting a superconformal point. (For more detailed discussion see appendix B.) Thus, it seems that the only gauge groups that 
can be realized on seven-branes in flux vacua for F-theory compactifications on $\mathcal{M}_{\max }$ will be subgroups of $G_{\max }$. The factors in $G_{\max }$ can generically be broken by nonzero G-flux components, however. An example of this was given in [14], where flux on the world-volume of a set of 7-branes on a divisor carrying a geometrically non-Higgsable $E_{8}$ gauge factor breaks the $E_{8}$ to $E_{7}$ in a setup with a clear heterotic dual geometry. More generally, we expect that $E_{8}$ factors can be broken to smaller groups. This seems like the most likely scenario for realizing the standard model through F-theory on $\mathcal{M}_{\max }$, by a G-flux breaking of $E_{8}$ to $\mathrm{SU}(3) \times \mathrm{SU}(2) \times \mathrm{U}(1)$, though other scenarios may be possible.

\subsection{The standard model and dark matter on $\mathcal{M}_{\max }$}

We do not attempt a thorough analysis here but make some comments on how the standard model may emerge and the likely nature of dark matter for F-theory compactifications on $\mathcal{M}_{\max }$.

Breaking $E_{8}$ to the standard model gauge group $\mathrm{SU}(3) \times \mathrm{SU}(2) \times \mathrm{U}(1)$ through fluxes may occur in a variety of ways. Some exploration of the possibility of realizing the standard model gauge group by breaking an $E_{8}$ without using Wilson lines was described in [34]. It would clearly be of interest to analyze such G-flux induced breakings more systematically in this context. $E_{8}$ can also be broken to the standard model group by first breaking to a unification group such as $\mathrm{SU}(5)$ or $\mathrm{SO}(10)$ and then breaking further through fluxes to $\mathrm{SU}(3) \times \mathrm{SU}(2) \times \mathrm{U}(1)$, potentially connecting with the methods used in F-theory GUT models [35-37].

As mentioned above, the details of how the standard model can arise from a flux breaking of $E_{8}$ in an F-theory construction are not fully understood. It seems, however, that even if such a construction is relatively unlikely compared to other breakings, this will impose only a small penalty on the overall weighting of the number of flux vacua. In particular, for the case of $\mathcal{M}_{\max }$ a flux configuration is characterized by $\chi / 24 \approx 75,000$ units of flux distributed among $b_{4} \approx 1,800,000$ possible cycles. Thus, a typical individual cycle will be given one unit of flux roughly $1 / 24$ of the time, two units of flux roughly $1 / 576$ of the time, etc.. Because the number of configurations is so large, a simple statistical model may suffice for estimating the fraction of models on $\mathcal{M}_{\max }$ that have a given breaking pattern for $G_{\max }$

As discussed in [38-41], the space of the 4-form flux can be divided into horizontal, vertical and the remaining components:

$$
H^{4}=H_{H *}^{4} \oplus H_{V *}^{2,2} \oplus H_{R M *}^{2,2} .
$$

The horizontal component of the flux does not break any gauge symmetry, and the rest of the components may break the gauge symmetry. For the Calabi-Yau fourfold in the regime of $h^{1,1} \ll h^{3,1}$, the size of the spaces $H_{V *}^{2,2} \oplus H_{R M *}^{2,2}$ are on the order of $h^{1,1}$, hence they are negligible compared to $H_{H *}^{4}$ (as found, for example, for the elliptically fibered Calabi-Yau fourfolds mentioned in [41]).

An important class of 4-cycles relevant for breaking a given $E_{8}$ factor are those that carry the "Cartan flux" $G=F \wedge \nu \in H^{2,2}[36,37,42]$. Here $F \in H^{1,1}$ is the 2-form dual 
to the divisor carrying $E_{8}$, and $\nu \in H^{1,1}$ is the 2-form dual to the exceptional divisor in a resolution of this Calabi-Yau fourfold.

For a given gauge group, the number of exceptional divisors is equal to its rank. If, for example a given breaking pattern of the $E_{8}$ requires that four specific cycles get units of flux, breaking the rank to 4 , this is suppressed by a factor ${ }^{1}$ of order $10^{6}$. Obviously, further specific choices may be needed to get exactly the standard model physics, but the tuning involved is unlikely to approach the level of one part in $10^{3000}$, which would be necessary for flux vacua from any other base to become relevant in the standard statistical picture.

Regarding dark matter, due to the diffuse nature of the fluxes, it seems that many of the additional factors in the gauge group (2.14) will either be unbroken, or broken only slightly by fluxes. So we would expect that this model predicts about 30 independent decoupled dark sectors, each with its own nonabelian gauge group of $E_{8}, F_{4}, G_{2} \times \mathrm{SU}(2)$ or subgroup thereof. Thus, in this scenario most dark matter would not be directly coupled to the standard model. While this might help explain the absence so far of a clear dark matter signal, it means that rather indirect means must be used to get a quantitative test of the validity of this hypothesis. A detailed analysis of the potential behavior of decoupled dark matter sectors of the form suggested here, such as a dark $G_{2} \times \mathrm{SU}(2)$ sector might shed light on what kind of observable features such dark sectors might give rise to. Note that additional dark matter such as an LSP or axions may arise from breaking of the $E_{8}$, supersymmetry breaking, and the stabilization of the moduli through the flux-generated superpotential.

\section{Distribution of flux vacua}

We now discuss the distribution of flux vacua and the reasons why we believe that other F-theory vacua are strongly suppressed relative to those on $\mathcal{M}_{\max }$.

\subsection{Flux vacua on $\mathcal{M}_{\max }$}

The counting problem of flux vacua was addressed in [5, 43-45], building on earlier insights from [46]. These methods were applied recently in the F-theory context in [40, 41, 47]. Consider a fourfold $X$ with $Q=\chi(X) / 24$ and fourth Betti number $b_{4} \approx \chi(X) \gg Q$. The problem of counting vacua can be related to the simplified problem of counting the number of lattice points in a $b_{4}$ dimensional sphere of radius $\sqrt{2 Q}$. In this regime, the volume of this high dimensional sphere is not a good approximation. Instead, using the method of $[5,48]$, we can estimate the total number of lattice points as follows:

The exact number of lattice points in this sphere is equal to

$$
N\left(b_{4}, Q\right)=\frac{1}{2 \pi i} \int \frac{d t}{t} e^{-Q t} Z(t)
$$

\footnotetext{
${ }^{1}$ Considering that there are $9 E_{8}$ gauge groups, this possibility is really of order $10^{-5}$.
} 
where the contour is along the imaginary axis and passes the pole $t=0$ on the left. (Note that with these conventions the integral runs from $i \infty$ to $-i \infty$.)

$$
Z(t)=\sum_{\vec{n} \in \mathbb{Z}^{b}} e^{t \vec{n}^{2} / 2}=\left(\sum_{n \in \mathbb{Z}} e^{t n^{2} / 2}\right)^{b} \equiv \vartheta_{3}\left(0, e^{t / 2}\right)^{b},
$$

where $\vartheta_{3}$ is the Jacobi theta function.

When $b$ is large, this integration can be evaluated by saddle point approximation:

$$
N\left(b_{4}, Q\right) \approx e^{S\left(t_{*}\right)}
$$

where $t_{*}$ is the point where $S(t)=-\ln (-t)-Q t+b \ln \vartheta_{3}\left(0, e^{t / 2}\right)$ takes an extremal value.

For our case $Q \approx b / 24, t_{*}=-6.18$ and

$$
N\left(b_{4}, Q\right) \sim 10^{3.59 \times Q}
$$

In the regime of $h^{1,1} \ll h^{3,1}, h^{2,1}=0$, the number of flux vacua is approximately

$$
N\left(h^{3,1}\right) \sim 10^{0.9 \times h^{3,1}}
$$

Applying this analysis to $\mathcal{M}_{\max }$, the total number of flux vacua is estimated to be of order $10^{272,000}$. Now, even if we only turn on the flux in $H_{H *}^{4}$ which does not break any gauge symmetry, since the dimension of $H_{V *}^{2,2} \oplus H_{R M *}^{2,2}$ is of order $h^{1,1} \sim 250$, the total number of flux vacua is only suppressed by a factor of $10^{50}$.

This analysis assumes among other things that every choice of flux associated with a set of $b$ independent integers gives rise to a solution in complex structure moduli space associated with a vacuum where the flux is self-dual. It is not clear that this is indeed the case. To get some sense of how this kind of condition may affect the number of vacua, we can consider another ensemble of vacua given by the set of $G_{4}$ flux that obey the self-duality relation with a fixed duality condition, where the number of independent fluxes giving the dimension of the sphere becomes $b_{4} / 2$, so that we need to evaluate $N\left(b_{4} / 2, Q\right)$.

In this case the saddle point approximation gives $t_{*}=-4.61$ and

$$
N\left(b_{4} / 2, Q\right) \sim 10^{2.95 \times Q}
$$

hence approximately

$$
N^{\prime}\left(h^{3,1}\right) \sim 10^{0.74 \times h^{3,1}} .
$$

Applying this formula to $\mathcal{M}_{\max }$, the total number of flux vacua $10^{224,000}$. While we use the formula (3.5) as an estimate for the number of flux vacua in this paper, it must be kept in mind that this is a very rough approximation that depends on assumptions about the solution space. For a realistic estimate of the number of vacua, a much more detailed analysis for this particular manifold would need to be carried out. 


\subsection{Suppression of other F-theory compactifications}

After $\mathcal{M}_{\max }$, the known fourfold with the next largest value of $h^{3,1}$ has $h^{1,1}=253, h^{3,1}=$ 299707, $\chi=1799808$. This fourfold is again a generic elliptic fibration over a threefold $B$ that is a $B_{2}$ bundle over $\mathbb{P}^{1}$, with a different choice of "twist". ${ }^{2}$ By the methods of estimation in the previous section, the number of flux vacua on this threefold is suppressed by a factor of roughly $\mathcal{O}\left(10^{-3000}\right)$ relative to those on $\mathcal{M}_{\text {max }}$. Further known fourfolds have numbers of flux vacua that rapidly become exponentially smaller, and the total number of known or inferred Calabi-Yau fourfolds is well below $10^{100}$, so known fourfolds cannot compete with the set of flux vacua on $\mathcal{M}_{\max }$. Note that the mirror to $\mathcal{M}_{\max }$ also has the same value of $\chi$, but a smaller value of $b_{4}=1,214,150$, giving a number of flux vacua smaller by a factor much smaller than $\mathcal{O}\left(10^{-3000}\right)$.

It is natural to wonder then whether there are as yet unknown Calabi-Yau fourfolds that can give numbers of flux vacua that dwarf the number of vacua on $\mathcal{M}_{\text {max }}$. Since there is not even a finiteness proof for elliptic Calabi-Yau fourfolds, this is certainly an open question. We briefly describe, however, some circumstantial evidence and analogies with the threefold case that suggest that $\mathcal{M}_{\text {max }}$ indeed contributes the lion's share of $4 \mathrm{~d}$ F-theory flux vacua and that there are no other individual vacua that compete, and further that there are also not enough other elliptic fourfolds to make a significant contribution even when all are added together.

First, we address the question of whether $\mathcal{M}_{\max }$ really contributes more flux vacua than any other individual elliptic CY fourfold. For example, could there exist another elliptic CY fourfold with a much larger value of $h^{3,1}$ ? While we do not have any way of proving that this does not occur, the corresponding situation for elliptic threefolds suggests that $\mathcal{M}_{\text {max }}$ really is the elliptic CY fourfold with the largest $h^{3,1}$. We review briefly the situation for threefolds. For elliptic Calabi-Yau threefolds, base surfaces can be classified using the minimal model approach to surfaces (see, e.g., [49]). It was shown by Grassi [50] that the only minimal surfaces that support elliptic threefolds are $\mathbb{P}^{2}$, the Hirzebruch surfaces $\mathbb{F}_{m}, 0 \leq m \leq 12$ and the Enriques surface. Combined with the structure of non-Higgsable clusters on base surfaces [13], this gives a systematic approach to constructing all allowed bases for elliptic Calabi-Yau threefolds [22, 28-30]. The largest possible value of $h^{2,1}$ for any elliptic Calabi-Yau threefold is proven to be 491, for the generic elliptic threefold over $\mathbb{F}_{12}$ [23]; this threefold was constructed using toric methods in the full analysis of Kreuzer and Skarke [51] and is indeed the Calabi-Yau threefold with the largest known value of $h^{2,1}$. The systematic analysis of all toric bases [22] and non-toric bases supporting elliptic threefolds with $h^{2,1} \geq 130$ [30] has shown that the simple toric hypersurface construction following Batyrev $[52,53]$ produces a large fraction of all possible bases that support elliptic threefolds, and that the toric construction is particularly effective in the region of large Hodge numbers.

Analogizing these results from threefolds to fourfolds, it seems quite plausible that the toric approach is also effective in constructing fourfolds with large $h^{3,1}$ (just as $h^{2,1}$ parameterizes complex structure for Calabi-Yau threefolds, $h^{3,1}$ plays the same role for

\footnotetext{
${ }^{2}$ Thanks to Y. Huang for discussions on this point.
} 
fourfolds). Thus, given that several approaches including the straightforward toric hypersurface method have produced the fourfold with $h^{3,1}=303,148$, which fits into the Hodge shield of fourfolds in a parallel way to the $h^{2,1}=491$ Calabi-Yau threefold for threefolds (see figure 1 and figure 2), it seems plausible that there will be no elliptic fourfolds with larger $h^{3,1}$. Another approach to this is to consider the generalization of the minimal model program to threefolds. While this story involves Mori theory [54] and is much more complicated in various ways, at a rough level we expect the minimal threefolds supporting elliptic Calabi-Yau fourfolds to be one of three types: Fano threefolds, $\mathbb{P}^{1}$ bundles (or more general conic bundles) over complex surfaces $B$, and bundles of a complex surface $B$ over $\mathbb{P}^{1}$. Fano threefolds that have been studied in this context have relatively small Hodge numbers [55]. A systematic analysis of $\mathbb{P}^{1}$ bundles over toric surfaces $B_{2}$ was carried out in [17], and none of these has a value of $h^{3,1}(X)$ that approaches 300,000. In the following section we describe an analysis of $B_{2}$ bundles over $\mathbb{P}^{1}$ that shows that at least for toric $B_{2}$, no such threefold gives a generic elliptic Calabi-Yau fourfold with larger $h^{3,1}(X)$ than $\mathcal{M}_{\max }$. Note that $B_{\max }$ is minimal in the class of smooth toric threefolds in the sense that no ray can be blown down to give another smooth toric threefold. Any blow-up of $B_{\max }$ on one or more curves or points gives a fourfold with lower $h^{3,1}(X)$, which can be described through a singular Weierstrass model on $B_{\max }$ with $(f, g)$ vanishing to orders $(4,6)$ on curves and $(8,12)$ at points.

Now we turn to the question of whether there are enough other elliptic Calabi-Yau fourfolds that even if each is suppressed by a factor much smaller than $\mathcal{O}\left(10^{-3000}\right)$ the total number of flux vacua from all other fourfolds might compete with the number on $\mathcal{M}_{\text {max }}$. As mentioned above, analysis of bases for elliptic threefolds shows that the number of non-toric bases is only larger than the number of toric bases by a relatively small overall factor, and toric bases are particularly well represented in the region of large $h^{2,1}$. In a separate work [18] we have recently carried out a Monte Carlo analysis of the set of toric threefolds that support elliptic Calabi-Yau fourfolds, including analysis of the Hodge numbers of the corresponding fourfolds and non-Higgsable structures on the threefolds bases. This Monte Carlo analysis shows that the number of toric threefold bases connected to $\mathbb{P}^{3}$ by blow-up and blow-down transitions without encountering $(4,6)$ curves is roughly $\mathcal{O}\left(10^{48}\right)$, and analogy with the $6 \mathrm{~d}$ case suggests that the complete set of toric threefold bases including cases with $E_{8}$ non-Higgsable gauge factors and $(4,6)$ curves is not much larger than this, and is probably well below $\mathcal{O}\left(10^{60}\right)$. This is a large number, but nowhere near enough to compete with the flux vacua on $\mathcal{M}_{\max }$, given that the number of flux vacua on each of these threefold bases is generally suppressed relative to $\mathcal{M}_{\max }$ by a factor much smaller than $\mathcal{O}\left(10^{-100,000}\right)$. Even if the number of non-toric threefold bases is much much larger than the number of toric bases, it is hard to imagine such a massive proliferation as would allow these bases to support fourfolds with flux compactifications that are as numerous as those on $\mathcal{M}_{\max }$, assuming as in the previous paragraph that each individual fourfold has a value of $h^{3,1}$ that is smaller than that of $\mathcal{M}_{\max }$ by thousands or tens of thousands.

We do not claim that we have proved anything here, certainly there are many more possible ways in which elliptic Calabi-Yau fourfolds may arise, and it is even possible 


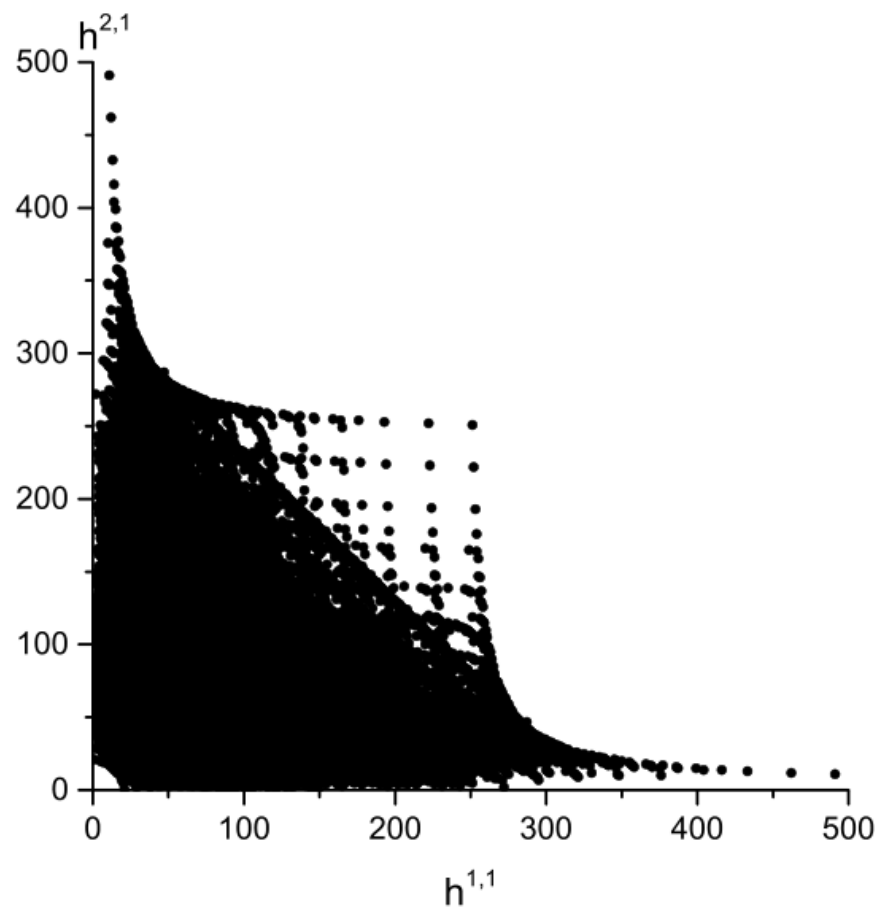

Figure 1. The distribution of $h^{1,1}$ and $h^{2,1}$ of the Calabi-Yau threefolds in the Kreuzer-Skarke database [51], corresponding to hypersurfaces in 4 dimensional reflexive polytopes. There is a clear "shield" structure and the Calabi-Yau threefold with largest $h^{2,1}$ is given by a generic elliptic fibration over $\mathbb{F}_{12}$.

that the number of such fourfolds may be infinite. But given the empirical information just summarized, this seems to us to be relatively unlikely, and it seems like the simplest situation would be that $\mathcal{M}_{\text {max }}$ indeed has the largest $h^{3,1}$ of a finite set of elliptic CalabiYau fourfolds that can be constructed as fibrations over fewer than e.g. $10^{100}$ bases, so that flux vacua on $\mathcal{M}_{\max }$ would dominate the set of F-theory vacua. Clearly, however, a more careful study of these questions is an important issue in verifying the general picture described in this paper.

\subsection{Other threefolds that are $B_{2}$ bundles over $\mathbb{P}^{1}$}

In this section we consider $B_{2}$ bundles over $\mathbb{P}^{1}$, where $B_{2}$ is a general smooth $2 \mathrm{~d}$ toric variety. Denote the $1 \mathrm{~d}$ rays in the fan of $B_{2}$ by $\left\{v_{i}\right\}(1 \leq i \leq N)$, then the $1 \mathrm{~d}$ rays of a $B_{2}$ bundle over $\mathbb{P}^{1}$ are given by:

$$
\begin{aligned}
w_{0} & =(0,0,1) \\
w_{i} & =\left(v_{i}, 0\right),(1 \leq i \leq N) \\
w_{N+1} & =\left(m v_{k}+n v_{k+1},-1\right),\left(m, n \geq 0,1 \leq k \leq N, v_{N+1} \equiv v_{1}\right) .
\end{aligned}
$$

The set of $3 \mathrm{~d}$ cones are given by $\left(w_{0}, w_{i}, w_{i+1}\right)$ and $\left(w_{N+1}, w_{i}, w_{i+1}\right)$ for $1 \leq i \leq(N-1)$, along with the cyclic case $\left(w_{0} / w_{N+1}, w_{N}, w_{1}\right)$. Hence the bundle is parametrized by a triple 


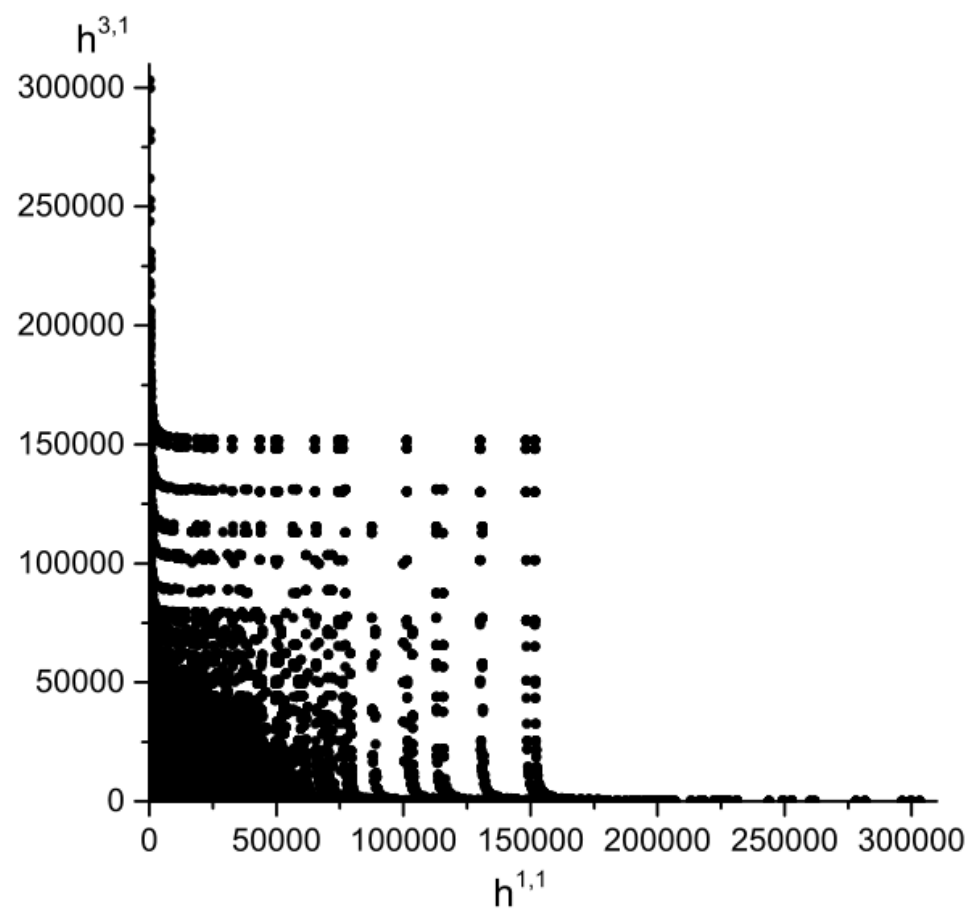

Figure 2. The distribution of $h^{1,1}$ and $h^{3,1}$ of the Calabi-Yau fourfolds in the Kreuzer-Skarke database [56], corresponding to hypersurfaces in 5 dimensional reflexive polytopes that are weighted projective spaces. There is also a clear "shield" structure and the Calabi-Yau fourfold with largest $h^{3,1}$ is $\mathcal{M}_{\max }$.

$(k, m, n)$. Now consider the set of monomials in $g$, which is given by the set of lattice points (called $A_{6}$ in appendix B)

$$
G=\left\{u \in \mathbb{Z}^{3} \mid \forall w_{i},\left\langle u, w_{i}\right\rangle \geq-6\right\} .
$$

For such a monomial $u \in G$, its order of vanishing on a toric divisor $w_{i}$ is equal to

$$
\operatorname{ord}_{w_{i}}(u \in G)=\left\langle u, w_{i}\right\rangle+6 .
$$

Its order of vanishing on the intersection of two toric divisors $w_{i}$ and $w_{j}$ is equal to

$$
\operatorname{ord}_{w_{i} w_{j}}(u \in G)=\left\langle u, w_{i}\right\rangle+\left\langle u, w_{j}\right\rangle+12 .
$$

Consider a monomial of the form $u=\left(u_{2}, z\right)$, where $u_{2} \in \mathbb{Z}^{2}$ is a $2 \mathrm{~d}$ vector. A necessary condition for $u \in G$ is

$$
\begin{aligned}
\left\langle u, w_{0}\right\rangle & \geq-6 \leftrightarrow z \geq-6 \\
\left\langle u, w_{N+1}\right\rangle & \geq-6 \leftrightarrow m\left\langle u_{2}, v_{k}\right\rangle+n\left\langle u_{2}, v_{k+1}\right\rangle-z \geq-6 .
\end{aligned}
$$

Hence for a given $u_{2}$, if there exists a monomial $u=\left(u_{2}, z\right) \in G$, the necessary condition is

$$
m\left\langle u_{2}, v_{k}\right\rangle+n\left\langle u_{2}, v_{k+1}\right\rangle \geq-12
$$


On the other hand, the absence of a $(4,6)$ singularity on the intersection of $w_{k}$ and $w_{k+1}$ requires that there exists a monomial $u=\left(u_{2}, z\right) \in G$, such that

$$
\left\langle u_{2}, v_{k}\right\rangle+\left\langle u_{2}, v_{k+1}\right\rangle \leq-7 .
$$

If $n=0, m \geq 13$, then from (3.13) we can see that $\left\langle u_{2}, v_{k}\right\rangle \geq 0$. Then because $\left\langle u_{2}, v_{k+1}\right\rangle \geq$ -6 , the condition (3.14) cannot be satisfied.

Following similar logic, the only possible pairs $(m, n)$ that satisfy these requirements are $(0,0 \sim 12),(1,1 \sim 6),(1 \sim 6,1)$ and $(0 \sim 12,0)$. Hence we only need to deal with a finite number of fibrations for each $B_{2}$.

The set of possible $B_{2}$ 's is precisely the set of 61,539 toric bases identified in [22] that support $6 \mathrm{~d}$ F-theory models. This is because if $B_{2}$ has a locus with where $(f, g, \Delta)$ vanish to orders $(4,6,12)$, any $B_{2}$ fibration over $\mathbb{P}_{1}$ also has such a locus and it is not allowed. A subtle issue arises when there are $(-9)-(-11)$ curves on $B_{2}$. In this case there are codimension two loci (points) on these curves where $(f, g)$ vanish to orders $(4,6)$. The solution is to blow up those points until they all become $(-12)$ curves. When a similar thing happens on a $3 \mathrm{~d}$ base, we assume such (non-toric) blow ups lead to a good base in F-theory.

To compute the Hodge number $h^{3,1}$ of a generic elliptically fibered Calabi-Yau fourfold over a $3 \mathrm{~d}$ base, we use the following approximate formula [26]:

$$
\begin{aligned}
h^{3,1} & \cong \tilde{h}^{3,1} \\
& =|F|+|G|-\sum_{\Theta \in \Delta, \operatorname{dim} \Theta=2} l^{\prime}(\Theta)-4+\sum_{\Theta_{i} \in \Delta, \Theta_{i}^{*} \in \Delta^{*}, \operatorname{dim}\left(\Theta_{i}\right)=\operatorname{dim}\left(\Theta_{i}^{*}\right)=1} l^{\prime}\left(\Theta_{i}\right) \cdot l^{\prime}\left(\Theta_{i}^{*}\right) .
\end{aligned}
$$

Here $\Delta^{*}$ is the convex hull of $\left\{w_{i}\right\}$ and $\Delta$ is the dual polytope of $\Delta^{*}$, defined to be

$$
\Delta=\left\{u \in \mathbb{R}^{3} \mid \forall v \in \Delta^{*},\langle u, v\rangle \geq-1\right\} .
$$

The symbol $\Theta$ denotes $2 \mathrm{~d}$ faces of $\Delta . \Theta_{i}$ and $\Theta_{i}^{*}$ denote the $1 \mathrm{~d}$ edges of the polytopes $\Delta$ and $\Delta^{*} \cdot l^{\prime}(\cdot)$ counts the number of integral interior points on a face. Here $F$ is the set $A_{4}$ in appendix $\mathrm{B}$, which counts the number of possible monomials in $f$.

After a thorough search among all the $B_{2}$ and triplets $(k, m, n)$ that specify a $B_{2}$ fibration, we have found that there is no elliptically fibered Calabi-Yau fourfold with $h^{3,1}>$ 303148 over any base threefold that is a toric $B_{2}$ bundle over $\mathbb{P}^{1}$. In fact, all the CY fourfolds with $h^{3,1} \gtrsim 236000$ arise from the following three $B_{2}$ bases:

$$
\begin{aligned}
& B_{a}=(-12 / /-12 / /-12 / /-12 / /-12 / /-12 / /-11 / /-11,6,0) \\
& B_{b}=(-12 / /-12 / /-12 / /-12 / /-12 / /-12 / /-12 / /-11 / /-12,6,0) \\
& B_{c}=(-12 / /-12 / /-12 / /-12 / /-12 / /-12 / /-11 / /-12,-1,5,0)
\end{aligned}
$$

The generic fibrations over $B_{a}, B_{b}$ and $B_{c}$ give elliptically fibered Calabi-Yau threefolds with Hodge numbers $\left(h^{1,1}, h^{2,1}\right)=(222,252),(251,251)$ and $(252,222)$ respectively. So they are all located near the self-dual point $(251,251)$ on the shield (see figure 1 ). We list the largest Hodge numbers $h^{3,1}$, that arise from constructing threefold bases as $B_{2}$ bundles over $\mathbb{P}^{1}$, using these $B_{2}$ bases, in table 1 . (Note that $B_{\max }$ is one of the $B_{b}$ bundles over $\mathbb{P}^{1}$.) 


\begin{tabular}{|c|c|c|}
\hline$B_{a}$ & $B_{b}$ & $B_{c}$ \\
\hline 261857 & $303148,299707,296266,292825$ & 261857 \\
258417 & $289384,285943,282502,281581$ & 258416 \\
254977 & $279061,278140,275620,274699$ & 254975 \\
251537 & $272179,271258,268738,267817$ & 251534 \\
248097 & $264376,261856,260935,260014$ & 248093 \\
244657 & $258415,257494,256573,254974$ & 244652 \\
243731 & $254053,253132,251533,250612$ & 243731 \\
241217 & $249691,248092,247171,246250$ & 241211 \\
237777 & $244651,243730,242809,241210$ & 237770 \\
236851 & $240289,239368,238447,237769$ & 236849 \\
& 236848 & \\
\hline
\end{tabular}

Table 1. List of the largest $h^{3,1}$ (greater than 236000) of generic elliptically fibered Calabi-Yau fourfolds over a base in the form of a $B_{a, b, c}$ bundle over $\mathbb{P}_{1}$.

Comparing to the largest values of $h^{3,1}$ appearing in the Kreuzer-Skarke database, it turns out that the values 243731, 261857, 278140, 281581, 299707 and 303148 in [56] all appear in table 1. Hence the known Calabi-Yau fourfolds with large $h^{3,1}$ are generally elliptically fibered. Since we also know that there are no non-toric base surfaces in the same region of the Hodge plot as $B_{b}$ [30], it seems reasonable to conclude that no $B_{2}$ bundle on $\mathbb{P}^{1}$ threefold base can give a fourfold with larger $h^{3,1}$ than $\mathcal{M}_{\max }$.

\section{Possible flaws in this scenario}

The idea that almost all solutions of string theory come from compactifications of F-theory on $\mathcal{M}_{\max }$ depends on quite a few assumptions, for which the level of evidence varies. In this section we discuss a variety of possible ways in which this scenario may break down. We begin with issues specific to the F-theory context and then discuss issues that arise in a more general framework.

The distribution. The Ashok-Denef-Douglas distribution of flux vacua (3.5) may be flawed or inaccurate. The precise distribution of flux vacua on $\mathcal{M}_{\max }$ or other F-theory models should be derived more explicitly. In particular, a more careful analysis is needed to determine precisely when fluxes can give SUSY solutions where the flux is self-dual.

Even within the context of F-theory it is not clear that all flux vacua on different bases should be weighted equally. There are several different types of transitions between different vacua, which may occur through tunneling processes that are wildly different and may cause different distributions to arise dynamically. In particular, there are Higgsing type transitions that involve movement on the geometric moduli space, which may be lifted by the superpotential, there are nonperturbative transitions between different flux vacua on the same base, and there are tensionless string type transitions between different bases. From the point of view of a random walk on the set of bases via blow-up and blow-down transitions as described in [18], the probability of reaching the base $B_{\max }$ is extremely low, 
and also involves many transitions through superconformal points associated with $(4,6)$ curves. So it is possible that dynamics may somehow make it difficult for the theory to reach this extreme point in the geometric moduli space of Calabi-Yau fourfolds. For a really sensible discussion of this kind of question, much more powerful tools and insight are needed.

Other fourfolds. We have outlined some arguments for why we do not expect other elliptic Calabi-Yau fourfolds with larger values of $h^{3,1}$, or enough other elliptic fourfolds with small values of $h^{3,1}$ to compete with flux vacua on $\mathcal{M}_{\max }$, but the evidence given is really only circumstantial, and it is possible that other, as yet unknown, elliptic Calabi-Yau fourfolds contribute more flux vacua than $\mathcal{M}_{\max }$.

Problems on $\mathcal{M}_{\max }$. Another possibility is that the set of flux vacua we have estimated on $\mathcal{M}_{\text {max }}$ may be wildly off because features specific to this geometry make most of the vacua inconsistent for one reason or another. For example, a superpotential from G-flux may push most vacua to an unphysical configuration, or one where an anthropically viable vacuum and/or the standard model may not be realizable. The detailed structure of flux vacua on $\mathcal{M}_{\max }$ should be studied in more detail to verify that these vacua indeed have sensible and potentially realistic physical structure.

Realizing the standard model. For observed physics to be realized in this scenario through F-theory on $\mathcal{M}_{\max }$ it seems that the standard model would need to be realized through flux breaking of $E_{8}$. The details of how this might work would need to be worked out, perhaps along the lines of [34], to confirm that this is a possible scenario. Two other possible ways of realizing the standard model in the F-theory context are through realizing part or all of the nonabelian part of the standard model gauge group through non-Higgsable clusters [15], or through tuning an F-theory SU(5) GUT model [36, 37]. While the nonabelian part of the standard model gauge group appears in F-theory for a wide range of threefold bases [18], it is not clear how or why the $\mathrm{U}(1)$ factor should arise in this scenario other than possible anthropic reasons. The $\mathrm{SU}(3) \times \mathrm{SU}(2)$ non-Higgsable product also does not appear on the bases with the largest $h^{3,1}$. As we have discussed, it is also not possible to tune an $\mathrm{SU}(5)$ on the bases with large $h^{3,1}$ and many flux vacua, and even in other bases, as discussed in [41] it requires tuning many moduli and is heavily suppressed in a natural distribution of flux vacua. Thus, challenges remain to see how any of the approaches to realizing the standard model will work in detail and play a dominant role in F-theory.

Other string constructions. From our current understanding, F-theory seems to provide larger classes of flux vacua than type IIB string theory. Our current understanding of flux vacua in other approaches to string compactification is less complete; it is possible that other constructions such as M-theory on $G_{2}$ manifolds, infinite families of IIA flux compactifications with large populations at relatively small compactification scales, non-geometric compactifications, or other approaches may give even more numerous flux compactifications than those of $\mathrm{F}$-theory on $\mathcal{M}_{\max }$. This discussion may at least motivate a specific 
search for such compactifications, and a more systematic approach to understanding and classifying the numbers of vacua in these other constructions.

Supersymmetry and supersymmetry breaking. In this paper we have focused on string vacuum solutions constructed through F-theory compactifications on Calabi-Yau fourfolds. Such geometries are expected to be associated with theories that preserve supersymmetry (SUSY) at some energy scale below the Planck scale. While supersymmetry has not yet been observed in nature, it is suggested by some aspects of observed physics (the hierarchy problem, gauge unification, possible dark matter candidates, etc.), and provides powerful theoretical constraints that allow us to make analytic progress in studying large classes of string vacua. For supersymmetric string vacuum constructions and the results described here to be relevant to "real-world" physics, either supersymmetry must be realized at some intermediate energy scale between the $\mathrm{TeV}$ scale and the Planck scale, or the qualitative results found here, or an analogue thereof, would have to be relevant for the class of string vacua where SUSY is broken at the Planck/compactification scale.

Of course, it is possible that supersymmetry does not hold below the Planck or compactification scale. In this case new methods are needed to analyze string compactifications without supersymmetry; for example, F-theory would need to be expanded to describe compactifications on elliptically fibered fourfolds without Calabi-Yau structure. It is possible that considerations analogous to those here may give rise to specific geometries and/or vacuum solutions without supersymmetry that still have specific structure analogous to the non-Higgsable gauge groups of $\mathcal{M}_{\text {max }}$, but much more work would be needed to make any general statements of this type.

From the long list of possible issues here, it is clear that much work remains to be done to confirm or rule out the scenario presented here in which most solutions of string theory with low-energy SUSY would arise from F-theory compactifications on $\mathcal{M}_{\max }$. This approach does, however, suggest a potential avenue by which string theory could provide a predictive framework for particle physics despite the enormous proliferation of vacua of the theory. While $10^{272,000}$ is a very large number of possible vacua, these vacua will all have many qualitative similarities, and the specific features of these vacua such as the subgroup of $G_{\max }$ preserved after flux breaking should be described by a calculable statistical distribution. Hopefully the specific scenario we have described here is sufficiently solid and compelling that it will stimulate further work, either to verify or to disprove this picture of how observable physics may arise naturally in string theory.

\section{Acknowledgments}

We would like to thank Lara Anderson, Andreas Braun, Will Detmold, James Gray, Jim Halverson, Yu-Chien Huang, Sam Johnson, David Morrison, Nikhil Raghuram, Ben Safdi, Sakura Schäfer-Nameki, Tracy Slatyer, and Frank Wilczek for helpful discussions. This research was supported by the DOE under contract \#DE-SC00012567. 


\section{A Linear transformation of the polytope containing $\mathcal{M}_{\max }$}

As mentioned in section 2, the elliptically fibered Calabi-Yau fourfold $\mathcal{M}_{\max }$ can be described as a hypersurface in a $5 \mathrm{~d}$ toric ambient space. This toric fivefold is a $\mathbb{P}^{2,3,1}$ bundle over the threefold base $B_{\max }$. The 3 d rays $w_{i}(0 \leq i \leq 100)$ in the toric fan of $B_{\max }$ are translated to the $5 \mathrm{~d}$ rays $\left(w_{i},-2,-3\right)$. In addition to that, there are two additional vertices: $(0,0,0,1,0)$ and $(0,0,0,0,1)$, which correspond to the $x$ and $y$ coordinates in the Weierstrass model $y^{2}=x^{3}+f x+g$ respectively.

The convex hull of the fan of the $5 \mathrm{~d}$ toric ambient space is a reflexive polytope $\tilde{\Delta}$ with the following 6 vertices:

$$
\begin{array}{lll}
V_{1}=(0,0,1,-2,-3), & V_{2}=(-1,-12,0,-2,-3), & V_{3}=(0,1,0,-2,-3), \\
V_{4}=(0,0,0,1,0), & V_{5}=(0,0,0,0,1), & V_{6}=(84,492,-1,-2,-3) .
\end{array}
$$

Its dual (polar) polytope (set of points $p$ satisfying $\left\langle p, V_{i}\right\rangle \geq-1$ for all $V_{i}$ ) $\Delta$ also has 6 vertices:

$$
\begin{array}{lll}
U_{1}=(78,-6,3606,-1,-1), & U_{2}=(35,-6,-6,-1,-1), & U_{3}=(-6,1,-6,-1,-1), \\
U_{4}=(0,0,0,2,-1), & U_{5}=(0,0,0,-1,1), & U_{6}=(78,-6,-6,-1,-1) .
\end{array}
$$

The toric fivefold in [27] which contains the Calabi-Yau fourfold $\mathcal{M}_{\max }$ is a weighted projective space $\mathbb{P}^{1,84,516,1204,1806}$. It is a (singular) toric variety with 6 vertices in the fan:

$$
\begin{array}{lll}
V_{1}^{\prime}=(1,0,0,0,0), & V_{2}^{\prime}=(0,1,0,0,0), & V_{3}^{\prime}=(0,0,1,0,0), \\
V_{4}^{\prime}=(0,0,0,1,0), & V_{5}^{\prime}=(0,0,0,0,1), & V_{6}^{\prime}=(-1,-84,-516,-1204,-1806) .
\end{array}
$$

There exists an $\mathrm{SL}(5)$ transformation matrix that transforms the $V_{i}$ into $V_{i}^{\prime}$ :

$$
\left(\begin{array}{ccccc}
0 & 0 & 1 & 0 & 0 \\
-1 & 0 & 0 & 0 & 0 \\
-12 & 1 & 0 & 0 & 0 \\
-26 & 2 & 2 & 1 & 0 \\
-39 & 3 & 3 & 0 & 1
\end{array}\right) \cdot\left(\begin{array}{cccccc}
0 & -1 & 0 & 0 & 0 & 84 \\
0 & -12 & 1 & 0 & 0 & 492 \\
1 & 0 & 0 & 0 & 0 & -1 \\
-2 & -2 & -2 & 1 & 0 & -2 \\
-3 & -3 & -3 & 0 & 1 & -3
\end{array}\right)=\left(\begin{array}{lllllc}
1 & 0 & 0 & 0 & 0 & -1 \\
0 & 1 & 0 & 0 & 0 & -84 \\
0 & 0 & 1 & 0 & 0 & -516 \\
0 & 0 & 0 & 1 & 0 & -1204 \\
0 & 0 & 0 & 0 & 1 & -1806
\end{array}\right) .
$$

\section{B The Weierstrass model on $B_{\max }$ and the possibility of tuning}

The set of monomials in the line bundle $\mathcal{O}(-n K)$ on $B_{\max }$ is given by the set

$$
A_{n}=\left\{u \in \mathbb{Z}^{3} \mid \forall w_{i},\left\langle u, w_{i}\right\rangle \geq-n\right\} .
$$

For such a monomial $u \in A_{n}$, its order of vanishing on a toric divisor $w_{i}$ is equal to

$$
\operatorname{ord}_{w_{i}}\left(u \in A_{n}\right)=\left\langle u, w_{i}\right\rangle+n
$$


A general Weierstrass model on $B_{\max }$ can be written in the following Tate form:

$$
y^{2}+a_{1} x y+a_{3} y=x^{3}+a_{2} x^{2}+a_{4} x+a_{6}
$$

The coefficients $a_{1} \in \mathcal{O}(-K), a_{2} \in \mathcal{O}(-2 K), a_{3} \in \mathcal{O}(-3 K), a_{4} \in \mathcal{O}(-4 K), a_{6} \in \mathcal{O}(-6 K)$. The order of vanishing of $a_{n}$ on a divisor $w_{i}$ is equal to

$$
\operatorname{ord}_{w_{i}}\left(a_{n}\right)=\left.\min \left(\left\langle u, w_{i}\right\rangle+n\right)\right|_{u \in A_{n}}
$$

The relations between these Tate coefficients and the functions $f$ and $g$ in the Weierstrass form:

$$
y^{2}=x^{3}+f x+g
$$

are $[57]$

$$
\begin{aligned}
& f=\frac{1}{48}\left(-\left(a_{1}^{2}+4 a_{2}\right)^{2}+24 a_{1} a_{3}+48 a_{4}\right) \\
& g=\frac{1}{864}\left(-\left(a_{1}^{2}+4 a_{2}\right)^{2}+36\left(a_{1} a_{3}+2 a_{4}\right)\left(a_{1}^{2}+4 a_{2}\right)-216\left(a_{3}^{2}+4 a_{6}\right)\right) .
\end{aligned}
$$

For a general elliptically fibered Calabi-Yau describe through Weierstrass model, the set of monomials in $f$ and $g$ are simply given by the sets $A_{4}$ and $A_{6}$ in (B.1).

The correspondence between the order of vanishing of those coefficients on a divisor $D$ and the gauge group on $D$ can be found in [57]. For example, if we want to tune an SU(5) gauge group on divisor $w_{i}$, then the order of vanishing of $\left(a_{1}, a_{2}, a_{3}, a_{4}, a_{6}\right)$ on $w_{i}$ should be $(0,1,2,3,5)$. This is impossible for a generic fibration on any base. However, we may remove a minimal number of points in $A_{n}$ (or equivalently, tune the coefficients of these monomials to be zero), so that this condition is satisfied. Since the order of vanishing will always increase after this tuning process, the value $\operatorname{ord}_{w_{i}}\left(a_{1}\right)$ should be equal to 0 before the tuning. The only divisors satisfying this constraint are

$$
w_{0}=(0,0,1), w_{1}=(-1,-12), w_{2}=(0,1), w_{100}=(84,492,-1) .
$$

However, after explicit computation, the tuning on these divisors always gives rise to codimension-1 locus with $(\operatorname{ord}(f), \operatorname{ord}(g))=(4,6)$. This codimension-1 singularity is nonminimal and not allowed in F-theory. Hence we cannot tune an $\mathrm{SU}(5)$ gauge group on $B_{\max }$.

A similar analysis can be applied for other gauge groups. It turns out that even the slightest enhancement of the Tate form (e.g. to $\mathrm{SU}(2)$ on some divisor) is not allowed on $B_{\max }$, due to the fact that $B_{\max }$ is a $B_{2}$ bundle over $\mathbb{P}^{1}$, and the base $B_{2}$ contains a lot of $-12,-1,-2,-2,-3,-1,-5,-1,-3,-2,-2,-1$ chains with "saturated" gauge groups.

Open Access. This article is distributed under the terms of the Creative Commons Attribution License (CC-BY 4.0), which permits any use, distribution and reproduction in any medium, provided the original author(s) and source are credited. 


\section{References}

[1] S. Weinberg, Anthropic bound on the cosmological constant, Phys. Rev. Lett. 59 (1987) 2607 [INSPIRE].

[2] WMAP collaboration, G. Hinshaw et al., Nine-Year Wilkinson Microwave Anisotropy Probe (WMAP) Observations: Cosmological Parameter Results, Astrophys. J. Suppl. 208 (2013) 19 [arXiv:1212.5226] [INSPIRE].

[3] M. Graña, Flux compactifications in string theory: a comprehensive review, Phys. Rept. 423 (2006) 91 [hep-th/0509003] [INSPIRE].

[4] M.R. Douglas and S. Kachru, Flux compactification, Rev. Mod. Phys. 79 (2007) 733 [hep-th/0610102] [INSPIRE].

[5] F. Denef, Les Houches Lectures on Constructing String Vacua, arXiv:0803.1194 [INSPIRE].

[6] O. DeWolfe, A. Giryavets, S. Kachru and W. Taylor, Type IIA moduli stabilization, JHEP 07 (2005) 066 [hep-th/0505160] [INSPIRE].

[7] B.S. Acharya and M.R. Douglas, A Finite landscape?, hep-th/0606212 [INSPIRE].

[8] C. Vafa, Evidence for F-theory, Nucl. Phys. B 469 (1996) 403 [hep-th/9602022] [InSPIRE].

[9] D.R. Morrison and C. Vafa, Compactifications of F-theory on Calabi-Yau threefolds. 1, Nucl. Phys. B 473 (1996) 74 [hep-th/9602114] [InSPIRE].

[10] D.R. Morrison and C. Vafa, Compactifications of F-theory on Calabi-Yau threefolds. 2., Nucl. Phys. B 476 (1996) 437 [hep-th/9603161] [INSPIRE].

[11] P. Candelas, E. Perevalov and G. Rajesh, Toric geometry and enhanced gauge symmetry of F-theory/heterotic vacua, Nucl. Phys. B 507 (1997) 445 [hep-th/9704097] [INSPIRE].

[12] M. Lynker, R. Schimmrigk and A. Wisskirchen, Landau-Ginzburg vacua of string, M-theory and F-theory at $c=12$, Nucl. Phys. B 550 (1999) 123 [hep-th/9812195] [InSPIRE].

[13] D.R. Morrison and W. Taylor, Classifying bases for 6D F-theory models, Central Eur. J. Phys. 10 (2012) 1072 [arXiv:1201.1943] [INSPIRE].

[14] L.B. Anderson and W. Taylor, Geometric constraints in dual F-theory and heterotic string compactifications, JHEP 08 (2014) 025 [arXiv: 1405.2074] [INSPIRE].

[15] A. Grassi, J. Halverson, J. Shaneson and W. Taylor, Non-Higgsable QCD and the Standard Model Spectrum in F-theory, JHEP 01 (2015) 086 [arXiv:1409.8295] [INSPIRE].

[16] D.R. Morrison and W. Taylor, Non-Higgsable clusters for 4D F-theory models, JHEP 05 (2015) 080 [arXiv:1412.6112] [INSPIRE].

[17] J. Halverson and W. Taylor, $P^{1}$-bundle bases and the prevalence of non-Higgsable structure in 4D F-theory models, JHEP 09 (2015) 086 [arXiv: 1506.03204] [INSPIRE].

[18] W. Taylor and Y.-N. Wang, A Monte Carlo exploration of threefold base geometries for $4 d$ F-theory vacua, arXiv:1510.04978 [INSPIRE].

[19] D.R. Morrison, TASI lectures on compactification and duality, hep-th/0411120 [INSPIRE].

[20] W. Taylor, TASI Lectures on Supergravity and String Vacua in Various Dimensions, arXiv:1104.2051 [INSPIRE].

[21] M.R. Douglas, The string landscape and low energy supersymmetry, arXiv:1204.6626. 
[22] D.R. Morrison and W. Taylor, Toric bases for 6D F-theory models, Fortsch. Phys. 60 (2012) 1187 [arXiv: 1204.0283] [INSPIRE].

[23] W. Taylor, On the Hodge structure of elliptically fibered Calabi-Yau threefolds, JHEP 08 (2012) 032 [arXiv:1205.0952] [INSPIRE].

[24] W. Fulton, Introduction to toric varieties. No. 131, Princeton University Press, Princeton U.S.A. (1993).

[25] V.I. Danilov. The geometry of toric varieties, Russian Math. Surv. 33 (1978) 97.

[26] A. Braun, W. Taylor and Y.N. Wang, to appear.

[27] P. Candelas and H. Skarke, F theory, SO(32) and toric geometry, Phys. Lett. B 413 (1997) 63 [hep-th/9706226] [inSPIRE].

[28] G. Martini and W. Taylor, 6D F-theory models and elliptically fibered Calabi-Yau threefolds over semi-toric base surfaces, JHEP 06 (2015) 061 [arXiv:1404.6300] [INSPIRE].

[29] S.B. Johnson and W. Taylor, Calabi-Yau threefolds with large $h^{2,1}$, JHEP 10 (2014) 23 [arXiv: 1406.0514] [INSPIRE].

[30] W. Taylor and Y.-N. Wang, Non-toric Bases for Elliptic Calabi-Yau Threefolds and 6D F-theory Vacua, arXiv:1504.07689 [INSPIRE].

[31] J.J. Heckman, D.R. Morrison and C. Vafa, On the Classification of 6D SCFTs and Generalized ADE Orbifolds, JHEP 05 (2014) 028 [Erratum ibid. 1506 (2015) 017] [arXiv: 1312.5746] [INSPIRE].

[32] M. Del Zotto, J.J. Heckman, D.R. Morrison and D.S. Park, 6D SCFTs and Gravity, JHEP 06 (2015) 158 [arXiv: 1412.6526] [INSPIRE].

[33] J.J. Heckman, D.R. Morrison, T. Rudelius and C. Vafa, Atomic Classification of $6 D$ SCFTs, Fortsch. Phys. 63 (2015) 468 [arXiv:1502.05405] [INSPIRE].

[34] L.B. Anderson, A. Constantin, S.-J. Lee and A. Lukas, Hypercharge Flux in Heterotic Compactifications, Phys. Rev. D 91 (2015) 046008 [arXiv:1411.0034] [INSPIRE].

[35] C. Beasley, J.J. Heckman and C. Vafa, GUTs and Exceptional Branes in F-theory - I, JHEP 01 (2009) 058 [arXiv: 0802.3391] [INSPIRE].

[36] C. Beasley, J.J. Heckman and C. Vafa, GUTs and Exceptional Branes in F-theory - II: Experimental Predictions, JHEP 01 (2009) 059 [arXiv: 0806.0102] [INSPIRE].

[37] R. Donagi and M. Wijnholt, Breaking GUT Groups in F-theory, Adv. Theor. Math. Phys. 15 (2011) 1523 [arXiv: 0808.2223] [inSPIRE].

[38] T.W. Grimm, T.-W. Ha, A. Klemm and D. Klevers, Computing Brane and Flux Superpotentials in F-theory Compactifications, JHEP 04 (2010) 015 [arXiv:0909.2025] [INSPIRE].

[39] N.C. Bizet, A. Klemm and D.V. Lopes, Landscaping with fluxes and the E8 Yukawa Point in F-theory, arXiv: 1404.7645 [INSPIRE].

[40] A.P. Braun and T. Watari, Distribution of the Number of Generations in Flux Compactifications, Phys. Rev. D 90 (2014) 121901 [arXiv:1408.6156] [InSPIRE].

[41] A.P. Braun and T. Watari, The Vertical, the Horizontal and the Rest: anatomy of the middle cohomology of Calabi-Yau fourfolds and F-theory applications, JHEP 01 (2015) 047 [arXiv: 1408.6167] [INSPIRE]. 
[42] A.P. Braun, A. Collinucci and R. Valandro, G-flux in F-theory and algebraic cycles, Nucl. Phys. B 856 (2012) 129 [arXiv:1107.5337] [INSPIRE].

[43] M.R. Douglas, The Statistics of string/M theory vacua, JHEP 05 (2003) 046 [hep-th/0303194] [INSPIRE].

[44] S. Ashok and M.R. Douglas, Counting flux vacua, JHEP 01 (2004) 060 [hep-th/0307049] [INSPIRE].

[45] F. Denef and M.R. Douglas, Distributions of flux vacua, JHEP 05 (2004) 072 [hep-th/0404116] [INSPIRE].

[46] R. Bousso and J. Polchinski, Quantization of four form fluxes and dynamical neutralization of the cosmological constant, JHEP 06 (2000) 006 [hep-th/0004134] [INSPIRE].

[47] T. Watari, Statistics of F-theory flux vacua for particle physics, JHEP 11 (2015) 065 [arXiv: 1506.08433] [INSPIRE].

[48] J.E. Mazo and A.M. Odlyzko, Lattice points in high-dimensional spheres, Monatsh. Math. 110 (1990) 47.

[49] W.P. Barth, K. Hulek, C.A.M. Peters and A. Van de Ven, Compact complex surfaces, Springer, Berlin Germany (2004).

[50] A. Grassi, On minimal models of elliptic threefolds, Math. Ann. 290 (1991) 287.

[51] M. Kreuzer and H. Skarke, Complete classification of reflexive polyhedra in four-dimensions, Adv. Theor. Math. Phys. 4 (2002) 1209 [hep-th/0002240] [INSPIRE]. Data available online at http://hep.itp.tuwien.ac.at/ kreuzer/CY/.

[52] V. Batyrev, Variations of the mixed Hodge structure of affine hypersurfaces in algebraic tori, Duke Math. J. 69 (1993) 349.

[53] V.V. Batyrev, Dual polyhedra and mirror symmetry for Calabi-Yau hypersurfaces in toric varieties, J. Alg. Geom. 3 (1994) 493 [alg-geom/9310003] [INSPIRE].

[54] K. Matsuki, Introduction to the Mori Program, Springer-Verlag, Berlin Germany (2002).

[55] A. Klemm, B. Lian, S.S. Roan and S.-T. Yau, Calabi-Yau fourfolds for M-theory and F-theory compactifications, Nucl. Phys. B 518 (1998) 515 [hep-th/9701023] [INSPIRE].

[56] M. Kreuzer and H. Skarke, Calabi-Yau four folds and toric fibrations, J. Geom. Phys. 26 (1998) 272 [hep-th/9701175] [INSPIRE].

[57] M. Bershadsky, K.A. Intriligator, S. Kachru, D.R. Morrison, V. Sadov and C. Vafa, Geometric singularities and enhanced gauge symmetries, Nucl. Phys. B 481 (1996) 215 [hep-th/9605200] [INSPIRE]. 